\title{
Krukenberg tumours from gastrointestinal cancers-analysis from a tertiary care centre in India
}

\author{
Srinivasan Muthukrishnan, Obla Lakshmanamoorthy Naganathbabu, Satish Devakumar Murugesan, \\ Ulagendra Perumal Srinivasan, Anbalagan Amudhan, Shanmugasundaram Rajendran
}

Institute of Surgical Gastroenterology, Rajiv Gandhi Government General Hospital \& Madras Medical College, Chennai, India

Contributions: (I) Conception and design: S Muthukrishnan; (II) Administrative support: OL Naganathbabu; (III) Provision of study material or patients: OL Naganathbabu, S Rajendran; (IV) Collection and assembly of data: S Muthukrishnan; (V) Data analysis and interpretation: S Muthukrishnan, S Devakumar, UP Srinivasan, A Amudhan; (VI) Manuscript writing: All authors; (VII) Final approval of manuscript: All authors. Correspondence to: Srinivasan Muthukrishnan. Institute of Surgical Gastroenterology, Rajiv Gandhi Government General Hospital \& Madras Medical College, Chennai 600003, India. Email: drsrim@gmail.com.

\begin{abstract}
Background: Krukenberg tumour (KT) is clinically defined as any ovarian metastatic carcinoma derived from a primary malignancy (usually from the gastrointestinal tract). Our aim was to analyse the incidence, demographic profile, clinical features, management and survival of patients with KTs from gastrointestinal tract malignancies at our centre.

Methods: This was a retrospective analysis data at our centre between Jan 2015 and Dec 2017.

Results: A total of 8 patients of KTs ( 2 from gastric and 6 from colorectal cancers) were observed with an incidence of $0.66 \%$ in gastric and $2.90 \%$ in colorectal cancers. The mean age of all the patients was 40 years (44 in gastric and 39 in colorectal group) and most of them (5 out of 8) occurred in premenopausal women. They were also most commonly bilateral (6 out of 8 ) and synchronous (6 out of 8). Surgical debulking was possible in 7 patients and all patients underwent systemic chemotherapy. Interestingly, 4 patients in the colorectal KT group with metastasis limited to ovary alone managed by surgical debulking and chemotherapy had a significantly higher 1-year survival compared to the others with peritoneal disease are alive at an average of 14.5 months.

Conclusions: The incidence of KTs from colorectal cancers is on the rise. KTs from colorectal cancers and KTs without peritoneal metastases have a better prognosis showing significantly higher 1-year survival rates following complete surgical resection and systemic chemotherapy.
\end{abstract}

Keywords: Krukenberg tumour (KT); colorectal; gastric; surgical debulking; 1-year survival

Submitted Jun 02, 2018. Accepted for publication Jul 03, 2018.

doi: 10.21037/jgo.2018.07.03

View this article at: http://dx.doi.org/10.21037/jgo.2018.07.03

\section{Introduction}

Krukenberg tumour (KT) refers to secondary malignant deposits in the ovary usually arising from gastrointestinal tract cancers. They are rare but pose a serious challenge in the management since the role of surgical debulking and chemotherapy and their effect on survival are controversial and still evolving. The aim of this study is to analyse the incidence, demographic profile, clinical features, management and survival of patients with KTs from gastrointestinal tract malignancy at our Institute.

\section{Methods}

This is a retrospective analysis of a prospectively maintained database at the Institute of surgical gastroenterology, Rajiv Gandhi Government General Hospital and Madras Medical College, Chennai, India between Jan 2015 to Dec 2017. Data of all patients with a diagnosis of KT from gastrointestinal tract malignancy were extracted. The 
Table 1 Characteristics of KTs in gastric and colorectal groups

\begin{tabular}{lccc}
\hline Variables & Gastric $(\mathrm{n}=2)$ & Colorectal $(\mathrm{n}=6)$ & Total $(\mathrm{n}=8)$ \\
\hline Incidence & $0.66 \%(2 / 301)$ & $2.90 \%(6 / 207)$ & $1.57 \%(8 / 508)$ \\
Mean age (years) & 44 & 39 & 50 \\
Premenopausal, $\mathrm{n}(\%)$ & $2(100.0)$ & $3(50.0)$ & $2(25.0)$ \\
Metachronous, $\mathrm{n}(\%)$ & $0(0.0)$ & $2(33.3)$ (average duration: 18.5 months) & 6 \\
Bilateral & 2 & 4 & 17.0 \\
Mean size (cm) & 8.5 & 4 & 4 \\
R0 resection, $\mathrm{n}$ & 0 & 6 & 4 \\
Chemotherapy, $\mathrm{n}$ & 2 & 4 & 4 \\
1-year survival, $\mathrm{n}$ & 0 & & 4 \\
\hline
\end{tabular}

$\mathrm{KT}$, Krukenberg tumour.

demographic details, clinical features, management and survival details were recorded. Analysis was done using SPSS version 20.

\section{Results}

A total of eight patients were diagnosed to have KTs secondary to GI malignancy (2 from gastric and 6 from colorectal primaries) (Table 1). The incidence of KT from gastric cancers was $0.66 \%$ ( 2 out of 301 cases) and that from colorectal cancers was $2.90 \%$ (6 out of 207 cases) during the study period. The mean age of the patients was 40 years overall (44 years in gastric and 39 years in the colorectal group). Five out of the 8 patients occurred in premenopausal women. While 6 of them were discovered synchronously, 2 patients had metachronous presentation, both from colonic primaries, 16 and 21 months after the primary surgery. Six out of the 8 cases had bilateral involvement of ovaries. The mean size of the ovarian tumour was $15 \mathrm{~cm}$ overall $(8.5 \mathrm{~cm}$ in the gastric group and $17.0 \mathrm{~cm}$ in the colorectal group).

The metastasis was limited to ovaries alone in 4 of the colorectal and none of the gastric cancer patients. Surgical removal of all macroscopic disease was possible in those four patients (Figure 1). In the remaining four patients ( 2 in colorectal group and 2 in gastric group) with peritoneal metastases, debulking of the ovarian tumour was done in three patients and diagnostic laparoscopy and biopsy alone was done in one patient in the gastric group with extensive peritoneal metastasis. All patients underwent systemic chemotherapy.

The mean follow-up was 12 months (range, 7-19 months).
It was found that the subgroup of four patients in colorectal group with metastasis limited to ovaries managed by surgical debulking and chemotherapy are alive at an average of 14.5 months and have a statistically significant $(\mathrm{P}=0.029)$ 1 -year survival compared to the other cases who had peritoneal involvement.

\section{Discussion}

$\mathrm{KT}$ is clinically defined as any ovarian metastatic carcinoma derived from a primary malignancy (usually from the gastrointestinal tract) (1). KTs are defined by the World Health Organization as ovarian carcinomas characterised by the presence of stromal involvement, mucin-producing neoplastic signet ring cells, and ovarian stromal sarcomatoid proliferation (1).

Literatures shows that about $76 \%$ of KTs originate from stomach, $11 \%$ in colorectum, $4 \%$ from the breast, $3 \%$ from the biliary system, $3 \%$ from the appendix, and the remaining 3\% from miscellaneous sites such as pancreas, uterine cervix, urinary bladder, or renal pelvis. However, recent articles have shown a higher incidence of KT of colorectal origin compared with those of gastric origin (2). In our series also, the incidence of KTs from colorectal primaries is much higher than from gastric primaries.

Prevalence of KT is higher in premenopausal patients than postmenopausal patients as observed in our series. It is postulated that the functioning ovary is prone to metastatic disease because the rich ovarian blood supply and leads to hematogenous metastasis (2). While most KTs present bilaterally, unilateral involvement is not uncommon and 

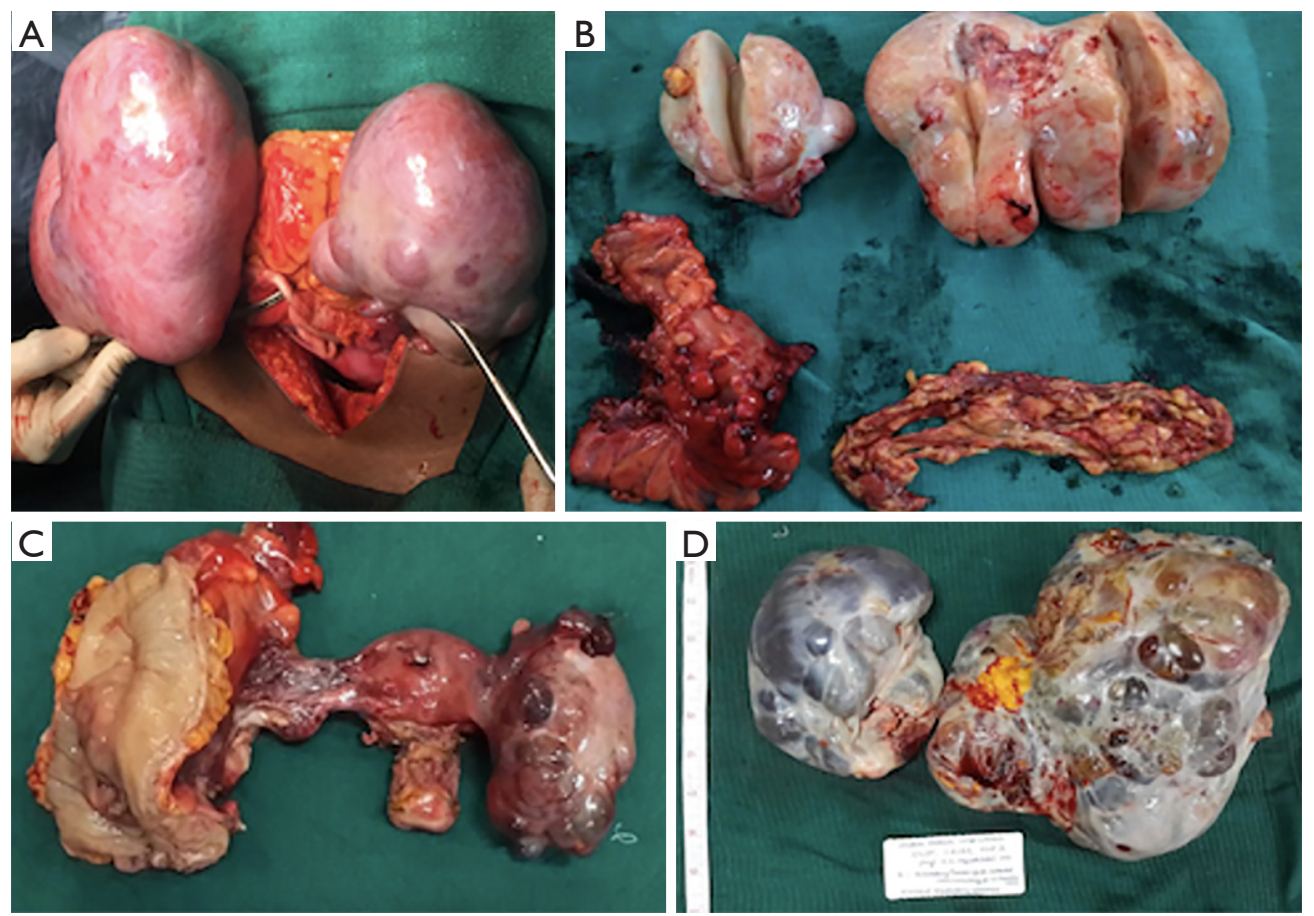

Figure 1 Operative specimen. (A) Sigmoid cancer with bilateral KT; (B) sigmoidectomy with b/l oophorectomy specimen; (C) Ca rectum with KT-posterior exenteration specimen; (D) metachronous KT s/p extended right hemicolectomy for Ca transverse colon. b/l, bilateral; Ca, carcinoma; s/p, status post; KT, Krukenberg tumour.

is more frequently seen in colorectal cancers. Moreover, in unilateral KTs, right ovary is more often involved than left one (2). In our series, there were two unilateral presentations and both were from colorectal cancers and were both in the right ovary.

Survival benefit of metastasectomy in metachronous KTs seems to be established by the most series. Survival analyses confirmed a statistically significant advantage for patients with no residual disease (R0) after resection compared to those with micro- or macroscopic residual disease (R1/R2) (3). In a recent paper Cho et al. (4) showed that metastasectomy plus chemotherapy offers superior overall survival when compared to palliative chemotherapy alone in gastric cancer with KT. Even in patients with peritoneal disease, when surgical resection is associated with hyperthermic intraperitoneal chemotherapy (HIPEC), patients with synchronous KTs presented a survival benefit with more than acceptable morbidity and mortality rates. The median survival for resected patients (both synchronous and metachronous) who underwent HIPEC procedure plus postoperative systemic CT was significantly longer with respect to patients who underwent just surgical procedure and postoperative systemic $\mathrm{CT}$ or just palliative $\mathrm{CT}$ after an explorative laparotomy (3).

Patients with KTs of colorectal origin showed more longer mean survival time than those of gastric origin especially in patients with synchronous, ascites positive, and ovary only metastasis. Achievement of complete cytoreduction, less extensive metastatic disease, and administration of systemic chemotherapy were all independently and strongly associated with improved overall survival (5). The American Society of Colon and Rectal Surgeons recommends that oophorectomy be performed in patients suspected or known to have ovarian involvement, either by direct extension or metastasis (6).

Prophylactic oophorectomy during surgery for colorectal cancer is currently not recommended. But molecular characterization of KTs can help in identifying the subset of patients with favourable prognosis and who will benefit from cytoreductive surgery, HIPEC, prophylactic bilateral ovariectomy, etc. (7). One study revealed that positive expression of three cancer stem cells (CSCs) related molecules, CD44, CD133 or Sox2, correlated with poor survival, and expression of Sox 2 was an independent 
prognostic factor in gastric KTs (8).

In our series, a subgroup of four patients in colorectal group with metastasis limited to ovaries managed by surgical debulking and chemotherapy had a statistically significant 1-year survival and are all alive at an average of 14.5 months, further reiterating the favourable prognosis of KTs from colorectal primaries.

\section{Conclusions}

KTs from gastrointestinal tract malignancies are not uncommon. Traditionally gastric cancer was the most common primary but recently, the incidence of KTs from colorectal cancers is on the rise. The incidence of KTs was found to be $0.66 \%$ in gastric and $2.9 \%$ in colorectal cancers in our study. They were also more common in premenopausal women and commonly bilateral in presentation. KTs from colorectal cancers and KTs without peritoneal metastases have a better prognosis showing significantly higher 1-year survival rates following complete surgical resection and systemic chemotherapy.

\section{Acknowledgements}

None.

\section{Footnote}

Conflicts of Interest: The authors have no conflicts of interest to declare.

Ethical Statement: The study was approved by Institutional Ethics Committee, Madras Medical College, Chennai (EC Reg No. ECR/270/Inst./TN/2013) and informed consent was taken from all the patients.

\section{References}

1. Qiu L, Yang T, Shan XH, et al. Metastatic factors for Krukenberg tumor: a clinical study on 102 cases. Med Oncol 2011;28:1514-9.

2. Jeung YJ, Ok HJ, Kim WG, et al. Krukenberg tumors of gastric origin versus colorectal origin. Obstet Gynecol Sci 2015;58:32-9.

3. Rosa F, Marrelli D, Morgagni P, et al. Krukenberg Tumors of Gastric Origin: The Rationale of Surgical Resection and Perioperative Treatments in a Multicenter Western Experience. World J Surg 2016;40:921-8.

4. Cho JH, Lim JY, Choi AR, et al. Comparison of Surgery Plus Chemotherapy and Palliative Chemotherapy Alone for Advanced Gastric Cancer with Krukenberg Tumor. Cancer Res Treat 2015;47:697-705. Erratum in: Cancer Res Treat 2018;50:302.

5. Xu KY, Gao H, Lian ZJ, et al. Clinical analysis of Krukenberg tumours in patients with colorectal cancer-a review of 57 cases. World J Surg Oncol 2017;15:25.

6. Chang GJ, Kaiser AM, Mills S, et al. Practice parameters for the management of colon cancer. Dis Colon Rectum 2012;55:831-43.

7. Kubeček O, Laco J, Špaček J, et al. The pathogenesis, diagnosis, and management of metastatic tumors to the ovary: a comprehensive review. Clin Exp Metastasis 2017;34:295-307.

8. Peng W, Hua RX, Jiang R, et al. Surgical treatment for patients with Krukenberg tumor of stomach origin: clinical outcome and prognostic factors analysis. PLoS One 2013;8:e68227.
Cite this article as: Muthukrishnan S, Naganathbabu OL, Murugesan SD, Srinivasan UP, Amudhan A, Rajendran S. Krukenberg tumours from gastrointestinal cancers-analysis from a tertiary care centre in India. J Gastrointest Oncol 2018;9(6):1164-1167. doi: 10.21037/jgo.2018.07.03 